\title{
AN UPDATED LIST OF Monogenoidea FROM MARINE FISHES OF VIETNAM
}

\author{
Nguyen Manh Hung*, Nguyen Van Ha, Ha Duy Ngo
}

Institute of Ecology and Biological Resources, VAST, Vietnam

Received 11 February 2020, accepted 16 April 2020

\section{ABSTRACT}

In this paper, we updated the list of monogenean species from marine fishes of Vietnam. Taxonomic position of monogenean species were arranged according to the current classification system. A total of 220 monogenean species from 152 marine fish species were listed. Distribution, hosts and references of each species were given. In addition, amendations of taxonomic status of taxa were also updated.

Keywords: Monogenoidea, marine fishes, East Sea, Gulf of Tonkin, Vietnam.

Citation: Nguyen Manh Hung, Nguyen Van Ha, Ha Duy Ngo, 2020. An updated list of Monogenoidea from marine fishes of Vietnam. Academia Journal of Biology, 42(2): 1-27. https://doi.org/10.15625/2615-9023/v42n2.14819.

*Corresponding author email: hung_iebr@yahoo.com

(C2020 Vietnam Academy of Science and Technology (VAST) 


\section{INTRODUCTION}

The study of monogeneans from marine fishes in Vietnam began in the 1950s when few intensive surveys were undertaken by the cooperation between Vietnam and Soviet Union parasitologists (Bychowsky \& Nagibina, 1954, 1959). The results of these studies were published by the Soviet helminthologists in Russian between 1961-1989 in a series of more than 30 papers. In addition, a large number of new genera and species were descripted and constitute much of the basis of our knowledge of monegenea fauna of marine fish in Vietnam and Southeast Asia. In 2006, Arthur \& Bui listed 55 monogenetic species from marine fishes. However, the list did not count many species which are present in some documents, such as Bychowsky \& Nagibina (1954, 1959, 1967, 1968, 1969a, 1969b, 1971, 1974, 1975a, 1975b, 1976, 1977), Bychowsky et al. (1976), Gusseve (1967, 1973, 1985), Timofeeva (1989), Egorova \& Korotaeve (1990), Egorova (2000). Moreover, many publiacations after 2006, e.g. Vo et al. (2010), Nguyen (2012), Dang et al. (2010, 2013), Dmitrieva et al. (2018), Kritsky et al. (2016) and Nguyen et al. (2016), added 35 species for monogenetic fauna in Vietnam.

This paper updated all monogenean species from marine fishes of Vietnam and arranged them to the current classification system.

\section{MATERIALS AND METHODS}

A total of 83 references relating to monogeneans from marine fishes of Vietnam were retrieved for this study. The taxa of monogeneans in the results part below were arranged following the classification system which was proposed by Bychowsky (1937) and revised by Boeger \& Kritsky (1993). The scientific name of monogeneans was checked and corrected according to the database of the World register of marine species (WoRMS, 2020). Similarly, the scientific name of marine fish hosts was updated following the database of the FishBase (https://www.fishbase.in/ search.php). In the list, the taxon levels, i.e. subfamilies, genera and species, were arranged in alphabetical order.

\section{RESULTS}

A total of 220 monogenetic species were found from 152 marine fish species. These monogeneans are belonging to 108 genera, 24 families, 5 orders, and 2 subclasses. The list of monogeneans below contains information about its taxonomical position, host species and distribution, and reference sources.

CLASS

MONOGENOIDEA BYCHOWSKY, 1937

SUBCLASS

BYCHOWSKY, 1937

Order CAPSALIDEA Lebedev, 1988

Family Capsalidae Baird, 1853

Genus Allobenedenia Yamaguti, 1963

1. A. epinepheli (Bychowsky \& Nagibina, 1967) Yang, Kritsky \& Sun, 2004

Syn. Megalocotyloides epinepheli Bychowsky \& Nagibina, 1967

Bychowsky \& Nagibina, 1967: 523; Vo et al., 2010: 39

Host: Malabar grouper Epinephelus malabaricus

Distribution: Gulf of Tonkin

2. A. yamagutii (Egorova, 1994) Yang, Kritsky \& Sun, 2004

Syn. Megalocotyloides yamagutiii Egorova, 1994

Vo et al., 2010: 39; Nguyen, 2012: 34

Host: Warsaw grouper Hyporthodus nigritus (syn. Epinephelus nigritus)* bay

Distributions: Nha Trang bay; Ha Long

Genus Benedenia Diesing, 1858

3. B. epinepheli (Yamaguti, 1937) Meserve, 1938

Vo et al., 2010: 38

Hosts: Duskytail grouper Epinephelus bleekeri, longtooth grouper E. bruneus; malabar grouper E. malabaricus, sixbar grouper $E$. sexfasciatus, greasy grouper $E$. 
tauvina, warsaw grouper Hyporthodus nigritus*

Distribution: Gulf of Tonkin

Genus Capsala Bosc, 1811 (Syn. Caballerocotyla Price, 1960)

4. C. affinis (Mamaev, 1968)

Syns. Caballerocotyla manteriaffinis Mamaev, 1968; Capsala manteriaffinis Chisholm \& Whittington, 2007

Mamaev, 1968: 9

Hosts: Kawakawa Euthynnus affinis; Atlantic bluefin tuna Thunnus thynnus

Distribution: Gulf of Tonkin

5. C. notosinense (Mamaev, 1968) Chisholm \& Whittington, 2007

Syn. Caballerocotyla notosinense Mamaev, 1968

Mamaev, 1968: 10

Host: Kawakawa E. affinis

Distribution: Gulf of Tonkin

6. C. paucispinosa (Mamaev, 1968) Chisholm \& Whittington, 2007

Syn. Caballerocotyla paucispinosa Mamaev, 1968

Mamaev, 1968: 12

Hosts: Kawakawa E. affinis, Atlantic bluefin tuna T. thynnus

Distribution: Gulf of Tonkin

Genus Encotyllabe Diesing, 1850

7. E. spari Yamaguti, 1934

Mamaev, 1970: 139

Host: Grey large-eye bream Gymnocranius griseus

Distribution: Gulf of Tonkin

Genus Megalocotyle Folda, 1928

8. M. Iutiani Lebedev, 1970

Lebedev, 1970: 193

Host: Bigeye snapper Lutjanus lutjanus

Distribution: Gulf of Tonkin

Genus Neobenedenia Yamaguti, 1963
9. N. melleni (MacCallum, 1927) Yamaguti, 1963

Vo et al., 2010: 41

Hosts: Duskytail grouper Epinephelus bleekeri, malabar grouper E. malabaricus, warsaw grouper Hyporthodus nigritus*

Distribution: Nha Trang bay

Genus Sessilorbis Mamaev, 1970

10. S. limopharynx Mamaev, 1970

Mamaev, 1970: 133

Host: Orbicular batfish Platax orbicularis

Distribution: Gulf of Tonkin

Genus Sprostoniella Bychowsky \& Nagibina, 1967

11. S. multitestis Bychowsky \& Nagibina, 1967

Bychowsky \& Naginina, 1967: 521

Host: Orbicular batfish P. orbicularis

Distributions: Gulf of Tonkin, South Cental coast

Genus Trilobiodiscus Bychowsky \& Nagibina, 1967 1967

12. T. lutiani Bychowsky \& Nagibina,

Bychowsky \& Naginina, 1967: 525

Host: Mangrove red snapper Lutjanus argentimaculatus

Distribution: Gulf of Tonkin

Genus Trochopus Diesing, 1850

13. T. antigoniae Egorova \& Korotaeva, 1990

Egorova \& Korotaeva, 1990: 443

Host: Indo-Pacific boarfish Antigonia rubescens

Distributions: Gulf of Tonkin, South Cental coast 1922

Family Dionchidae Johnston \& Tiegs,

Genus Dionchus Goto, 1899

14. D. bychowskyi Timofeeva, 1989

Timofeeva, 1989: 59 
Nguyen Manh Hung et al.

Host: Cobia Rachycentron canadum

Distribution: Gulf of Tonkin

15. D. major Egorova, 2000

Timofeeva, 2000: 257

Host: Cobia R. canadum

Distribution: Gulf of Tonkin

16. D. minor Timofeeva, 1989

Timofeeva, 1989: 62

Host: Live sharksucker Echeneis naucrates

Distribution: Gulf of Tonkin

17. D. nagibinae Timofeeva, 1989

Timofeeva, 1989: 64

Host: Cobia R. canadum

Distributions: Gulf of Tonkin, South Cental coast

18. D. orientalis Timofeeva, 1989

Timofeeva, 1989: 57

Host: Live sharksucker E. naucrates

Distribution: Gulf of Tonkin

19. D. sinensis Timofeeva, 1989

Timofeeva, 1989: 65

Host: Live sharksucker E. naucrates

Distribution: Gulf of Tonkin 1937

Order DACTYLOGYRIDEA Bychowsky,

\section{Family Calceostomatidae Parona \&} Perugia, 1890

Genus Bychowskya Nagibina, 1968

20. B. drepanae Nagibina, 1968

Nagibina, 1968: 289

Host: Spotted sicklefish Drepane punctate

Distribution: Gulf of Tonkin

Genus Dicrumenia Mamaev, 1969

21. D. bychowskyi Mamaev, 1969

Mamaev, 1969: 170

Host: Saddle grunt Pomadasys maculatus

Distribution: Gulf of Tonkin
1933

\section{Family Dactylogyridae Bychowsky,}

Genus Ancyrocephaloides Yamaguti, 1938

22. A. chauhani Bychowsky \& Nagibina, 1975

Bychowsky \& Nagibina, 1975a: 70

Host: Short-nosed tripodfish Triacanthus brevirostris

Distribution: Gulf of Tonkin

Genus Ancyrocephalus Creplin, 1839

23. A. macrogaster Yamaguti, 1953

Mamaev, 1970: 131

Host: Whipfin silver-biddy Gerres filamentosus

Distribution: Gulf of Tonkin

24. A. parspinicirrus Mamaev, 1970

Mamaev, 1970: 129

Host: Spotted sicklefish Drepane punctate

Distribution: Gulf of Tonkin

25. A. unicirrus Tripathi, 1959

Mamaev, 1970: 133

Host: Silver grunt Pomadasys hasta

Distribution: Gulf of Tonkin

Genus Atherinicus Bychowsky \& Nagibina, 1969

26. A. cornutus Bychowsky \& Nagibina, 1969

Bychowsky \& Nagibina, 1969b: 523

Host: Red Sea hardyhead silverside Atherina forskalii

Distribution: Gulf of Tonkin

Genus Bravohollisia Bychowsky \& Nagibina, 1970 1970

27. B. magna Bychowsky \& Nagibina,

Bychowsky \& Nagibina, 1970a: 22

Host: Silver grunt Pomadasys hasta

Distributions: Gulf of Tonkin, South Cental coast 

1970

28. B. pomadasis Bychowsky \& Nagibina,

Bychowsky \& Nagibina, 1970a: 21

Hosts: Silver grunt $P$. hasta; saddle grunt P. maculatus coast

Distribution: Gulf of Tonkin, South Cental

29. B. tecta Bychowsky \& Nagibina, 1970

Bychowsky \& Nagibina, 1970a: 20

Host: Saddle grunt $P$. maculatus

Distribution: Gulf of Tonkin, South Cental coast

Genus Caballeria Bychowsky \& Nagibina, 1970

30. C. pedunculata Bychowsky \& Nagibina, 1970

Bychowsky \& Nagibina, 1970a: 23

Host: Silver grunt P. hasta

Distributions: Gulf of Tonkin, South Cental coast

31. C. robusta Bychowsky \& Nagibina, 1970

Bychowsky \& Nagibina, 1970a: 24

Host: Saddle grunt $P$. maculatus

Distributions: Gulf of Tonkin, South Cental coast

Genus Chauhanellus Bychowsky \& Nagibina, 1969 1968

32. C. flexiosus Bychowsky \& Nagibina,

Bychowsky \& Nagibina, 1969a: 365

Host: Smooth-headed catfish Arius leiotetocephalus

Distributions: Gulf of Tonkin, South Cental coast

33. C. oculatus Bychowsky \& Nagibina, 1968

Bychowsky \& Nagibina, 1969a: 364

Host: Smooth-headed catfish leiotetocephalus
Distributions: Gulf of Tonkin, South Cental coast

Genus Dactylogyrus Diesing, 1850

34. D. gotoi Gussev, 1965

Gussev, 1967: 251

Host: Japanese seabass Lateolabrax japonicus

Distribution: Gulf of Tonkin

35. D. inversus Goto \& Kikuchi, 1917

Gussev, 1967: 251

Host: Japanese seabass L. japonicus

Distribution: Gulf of Tonkin

36. D. kikuchii Gussev, 1965

Gussev, 1967: 252

Host: Japanese seabass L. japonicus

Distribution: Gulf of Tonkin

Genus Diversohamulus Bychowsky \& Nagibina, 1969

37. D. tricuspidatus Bychowsky \& Nagibina, 1969

Bychowsky \& Nagibina, 1969b: 525

Host: Sumatran silverside Atherina bleeker

Distribution: Gulf of Tonkin 2002

Genus Euryhaliotrema Kritsky \& Boeger,

38. E. johni (Tripathi, 1959) Kritsky \& Boeger, 2002

Kritsky \& Boeger, 2002: 30; Nguyen, 2012: 28

Hosts: Blacktail snapper $L$. fulvus, bigeye snapper $L$. lutjanus, russell's snapper $L$. russelli; blackhead seabream Acanthopagrus schlegelii

Distribution: Ha Long bay 1922

Genus Haliotrema Johnston \& Tiegs,

39. H. ampliocuspidis Bychowsky \& A. Nagibina, 1971

Bychowsky \& Nagibina, 1971: 28 
Host: Common ponyfish Leiognathus equulus

Distribution: Gulf of Tonkin 1970

40. H. australe Bychowsky \& Naginina,

Bychowsky \& Naginina, 1970b: 1791

Host: Indian goatfish Parupeneus indicus

Distribution: Gulf of Tonkin

41. H. bilobatum (Yamaguti, 1953) Bychowsky \& Nagibina, 1970

Bychowsky \& Naginina, 1970b: 1794

Hosts: Warsaw grouper Hyporthodus nigritus*; spotted sicklefish Drepane punctate

Distribution: Gulf of Tonkin

42. H. cromileptis Young, 1968

Vo et al., 2010: 35

Host: Warsaw grouper H. nigritus*

Distribution: Gulf of Tonkin

43. H. epinepheli Young, 1968

Vo et al., 2010: 34

Hosts: Areolate grouper Epinephelus areolatus, yellow grouper E. awoara, duskytail grouper E. bleekeri, Hong Kong grouper E. akaara, sixbar grouper $E$. sexfasciatus; red seabream Pagrus major (Syn. Pagrosomus major); goldlined seabream Rhabdosargus sarba

Distribution: Gulf of Tonkin, South Cental coast

44. H. flagellocirrus Bychowsky \& Nagibina, 1970

Bychowsky \& Naginina, 1970b: 1794

Host: sweepers Pempheris molucca

Distribution: Gulf of Tonkin

45. H. geminatohamula Bychowsky \& Naginina, 1971

Bychowsky \& Nagibina, 1971: 1796

Host: Spotnape ponyfish N. nuchalis

Distribution: Gulf of Tonkin

46. H. holocentri Young, 1968

Bychowsky \& Nagibina, 1970b: 1793
Host: Redcoat Redcoat Sargocentron rubrum (syn. Holocentrus ruber)

Distribution: Gulf of Tonkin 1970

47. H. johsntoni Bychowsky \& Nagibina,

Bychowsky \& Nagibina, 1970b: 1798

Host: Freckled goatfish Upeneus tragula

Distribution: Gulf of Tonkin

48. H. magnihamus Bychowsky \& Nagibina, 1970

Bychowsky \& Nagibina, 1970b: 1797

Host: Spotted sicklefish Drepane punctate

Distribution: Gulf of Tonkin

49. H. neobilobatus Bychowsky \& Nagibina, 1970

Bychowsky \& Nagibina, 1970b: 1795

Host: Spotted sicklefish D. punctate

Distribution: Gulf of Tonkin

50. H. spinicirrus (Yamaguti, 1953) Bychowsky \& Nagibina, 1970

Syn. Ancyrocephalus spinicirrus Yamaguti, 1953

Mamaev, 1970: 129; Nguyen, 2012: 31

Hosts: Spotted sicklefish D. punctate; Japanese scad Decapterus maruadsi

Distribution: Gulf of Tonkin

51. H. subtilihamula Bychoswky \& Nagibina, 1971

Bychoswky \& Nagibina, 1971: 32

Host: Toothpony Gazza minuta

Distribution: Gulf of Tonkin

52. H. surculocirrus Bychowsky \& Nagibina, 1971

Bychoswky \& Nagibina, 1971: 34

Host: Striped ponyfish Aurigequula fasciata (syn. Leiognathus fasciatus)

Distribution: Gulf of Tonkin

53. H. parvihamus Bychowsky \& Nagibina, 1970

Bychowsky \& Naginina, 1970b: 38 
Host: Spotted sicklefish D. punctate

Distribution: Gulf of Tonkin 1970

54. H. pempherii Bychowsky \& Nagibina,

Bychowsky \& Naginina, 1970b: 36

Host: Sweepers Pempheris molucca

Distribution: Gulf of Tonkin

55. H. platycephali Yin \& Sproston, 1948

Nguyen, 2012: 30

Host: Bartail flathead Platycephalus indicus

Distribution: Gulf of Tonkin 1971

56. H. tenucirrus Bychowsky \& Nagibina,

Bychowsky \& Naginina, 1970b: 37

Host: Common ponyfish L. equulus

Distribution: Gulf of Tonkin 1970

57. H. tiegsi Bychowsky \& Nagibina,

Bychowsky \& Naginina, 1970b: 38

Host: Freckled goatfish U. tragula

Distribution: Gulf of Tonkin

Genus Haliotrematoides Kritsky, Yang \& Sun, 2009

58. H. calcaris (Bychowsky \& Nagibina, 1971) Kritsky, Yang \& Sun, 2009

Bychowsky \& Nagibina, 1971: 34

Host: snapper Lutjanus sp.

Distribution: Gulf of Tonkin

59. H. longitubocirrus (Bychowsky \& Nagibina, 1971) Kritsky, Yang \& Sun, 2009

Bychowsky \& Nagibina, 1971: 36

Hosts: Bigeye snapper L. lutjanus, brownstripe red snapper $L$. vitta

Distribution: Gulf of Tonkin

60. H. noncalcaris (Bychowsky \& Nagibina, 1971) Kritsky, Yang \& Sun, 2009

Bychowsky \& Nagibina, 1971: 35

Host: Mangrove red snapper $L$. argentimaculatus
Distribution: Gulf of Tonkin

61. H. patellacirrus (Bychowsky \& Nagibina, 1971) Kritsky, Yang \& Sun, 2009

Bychowsky \& Nagibina, 1971: 37

Hosts: Bigeye snapper L. lutjanus, dory snapper L. fulviflammus

Distribution: Gulf of Tonkin 1953

Genus Hamatopeduncularia Yamaguti,

62. H. arii Yamaguti, 1953

Bychowsky \& Nagibina, 1969a: 357

Host: Smooth-headed catfish Arius leiotetocephalus

Distribution: Gulf of Tonkin 1968

63. H. elegans Bychowsky \& Nagibina,

Bychowsky \& Nagibina, 1969a: 362

Host: Smooth-headed catfish A. arius

Distribution: Gulf of Tonkin 1969

64. H. simplex Bychowsky \& Nagibina,

Bychowsky \& Nagibina, 1969a: 361

Host: Smooth-headed catfish A. arius

Distribution: Gulf of Tonkin 1969

65. H. thalassini Bychowsky \& Nagibina,

Bychowsky \& Nagibina, 1969a: 361

Host: Smooth-headed catfish A. arius

Distribution: Gulf of Tonkin

Genus Hemirhamphiculus Bychowsky \& Nagibina, 1969 1969

66. H. armatus Bychowsky \& Nagibina,

Bychowsky \& Nagibina, 1969b: 518

Host: Black-barred halfbeak Hyporhamphus far (syn. Hemiramphus far)

Distribution: Gulf of Tonkin

67. H. similis Bychowsky \& Nagibina, 1969

Bychowsky \& Nagibina, 1969b: 519 
Nguyen Manh Hung et al.

$\begin{array}{lrr}\text { Host: } & \text { Dussumier's } & \text { halfbeak } \\ \text { Hyporhamphus } & \text { dussumieri } & \text { (syn. } \\ \text { Hemiramphus dussumieri) } & \\ \text { Distribution: Gulf of Tonkin } \\ \text { Genus Ligophorus Euzet \& Suriano, } 1977 \\ \text { 68. L. fenestrum Soo \& Lim, } 2012\end{array}$

Dmitrieva et al., 2018: 72

Hosts: Bluespot mullet Crenimugil seheli (syn. Moolgarda seheli), largescale mullet Planiliza macrolepis, otomebora mullet $P$. melinopterus, mullet Valamugil formosae, speigler's mullet $V$. speigleri

Distributions: Gulf of Tonkin; South Cental coast

69. L. hamulosus Pan et Zhang, 1999

Nguyen, 2012: 26

Hosts: bluespot mullet Crenimugil seheli; kanda Osteomugil engeli (syn. Valamugil engeli)

Distribution: Gulf of Tonkin

70. L. leporinus (Zhang \& Ji, 1981) Gussev, 1985

Gussev, 1985: 128; Nguyen, 2012: 28

Hosts: Bluespot mullet Crenimugil seheli, squaretail mullet Ellochelon vaigiensis, flathead grey mullet Mugil cephalus, kanda Osteomugil engeli, largescale mullet Planiliza macrolepis (syn. Liza macrolepis), eastern keelback mullet $P$. affinis (syn. L. affinis)

Distributions: Gulf of Tonkin; South Cental coast

71. L. macrocolpos Euzet \& Suriano, 1977

Te, 1998: 5; Arthur \& Bui, 2006: 46

Host: Flathead grey mullet Mugil cephalus

Distribution: Gulf of Tonkin

Genus Lobotrema Tripathi, 1959

72. L. sciaenae (Bychowsky \& Nagibina, 1977) Oliver, 1987

Syn. Allomurraytrema sciaennae Bychowsky \& Nagibina, 1977

Bychowsky \& Nagibina, 1977: 14
Hosts: Sin croaker Johnius dussumieri (syn. Sciaena dussumieri), belanger's croaker J. belengri, yellow drum Nibea albiflora

Distributions: Gulf of Tonkin; South Cental coast

73. L. argyrosomi (Bychowsky \& Nagibina, 1977) Oliver, 1987

Syn. Allomurraytrema argyrosomi Bychowsky \& Nagibina, 1977

Bychowsky \& Nagibina, 1977: 12

Host: Amoy croaker Argyrosomus amoyensis

Distributions: Gulf of Tonkin; South Cental coast

Genus Metahaliotrema Yamaguti, 1953

74. M. kulkarnii Venkatanarasaiah, 1981

Kritsky et al., 2016: 330

Host: Spotted scat Scatophagus argus

Distributions: Gulf of Tonkin; South Cental coast

75. M. mizellei Venkatanarasaiah, 1981

Nguyen, 2012: 33; Kritsky et al., 2016: 326

Host: Spotted scat S. argus Lan

Distributions: Gulf of Tonkin; vịnh Thái

76. M. scatophagi Yamaguti, 1953

Kritsky et al., 2016: 324

Host: Spotted scat S. argus

Distributions: Gulf of Tonkin; vịnh Thái Lan

77. M. similis Kritsky, Nguyen, Ha \& Heckman, 2016

Kritsky et al., 2016: 332

Host: Spotted scat S. argus

Distribution: Gulf of Tonkin 1964

78. M. cf. yamagutii Mizelle \& Price,

Kritsky et al., 2016: 333

Host: Spotted scat S. argus

Distribution: Gulf of Tonkin 
79. M. ypsilocleithrum Kritsky, Nguyen, Ha \& Heckman, 2016

Kritsky et al., 2016: 328

Host: Spotted scat S. argus Lan

Distributions: Gulf of Tonkin; vịnh Thái

Genus Paradiplectanotrema Gerasev, Gayevskaya \& Kovaleva, 1987

80. P. trachuri (Kovaljova, 1970) Gerasev, Gayevskaya \& Kovaleva, 1987

Nguyen, 2012: 34

Hosts: Japanese meagre Argyrosomus japonicus, caroun croaker Johnius carouna

Distribution: Gulf of Tonkin

Genus

Parahemirhamphiculus Bychowsky \& Nagibina, 1969 1969

81. P. pinguis Bychowsky \& Nagibina,

Bychowsky \& Nagibina, 1969b: 521

Hosts: Black-barred halfbeak Hyporhamphus far

Distribution: Gulf of Tonkin

Genus Platycephalotrema Kritsky \& Nitta, 2019

82. P. macassarense (Yamaguti, 1963) Kritsky \& Nitta, 2019

Bychowsky \& Nagibina, 1971: 38

Host: Bartail flathead Platycephalus indicus

Distributions: Gulf of Tonkin

83. P. thysanophrydis (Yamaguti, 1937) Kritsky \& Nitta, 2019

Bychowsky \& Nagibina, 1971: 39

Hosts: Japanese flathead Inegocia japonica (syn. Thysanophrys japonicus)

Distribution: Gulf of Tonkin

Genus Protogyrodactylus Johnston \& Tiegs, 1922 1974

84. P. alienus Bychowsky \& Nagibina,

Bychowsky \& Nagibina, 1974: 477
Hosts: Whipfin silver-biddy Gerres filamentosus; saddleback silver-biddy $G$. limbatus (syn. G. lucidus)

Distribution: Gulf of Tonkin 1974

85. P. delicatus Bychowsky \& Nagibina,

Bychowsky \& Nagibina, 1974: 478

Host: Saddleback silver-biddy G. limbatus

Distribution: Gulf of Tonkin 1974

86. P. gussevi Bychowsky \& Nagibina,

Bychowsky \& Nagibina, 1974: 473

Host: Jarbua terapon Terapon jarbua

Distribution: Gulf of Tonkin 1974

87. P. elegantis Bychowsky \& Nagibina,

Bychowsky \& Nagibina, 1974: 481

Host: Deep-bodied mojarra G. erythrourus

Distribution: Gulf of Tonkin 1974

88. P. fissilis Bychowsky \& Nagibina,

\section{Bychowsky \& Nagibina, 1974: 481}

Host: Slender silver-biddy G. oblongus (syn. G. macrosoma)

Distribution: Gulf of Tonkin

89. P. marinoides Bychowsky \& Nagibina, 1974

Bychowsky \& Nagibina, 1974: 476

Host: Jarbua terapon T. jarbua

Distribution: Gulf of Tonkin

90. P. marinus Gussev, 1973

Gussev, 1973: 17

Host: Jarbua terapon T. jarbua

Distribution: Gulf of Tonkin

91. P. perforatus Bychowsky \& Nagibina, 1974

Bychowsky \& Nagibina, 1974: 476

Host: Jarbua terapon T. jarbua

Distribution: Gulf of Tonkin 
Nguyen Manh Hung et al.

92. Protogyrodactylus scapulasser (Mamaev, 1970) Gussev, 1973

Syn. Ancyrocephalus scapulasser Mamaev, 1970

Mamaev, 1970: 131; Gussev, 1973: 23

Host: Whipfin silver-biddy $G$. filamentosus

Distribution: Gulf of Tonkin 1974

93. P. solidus Bychowsky \& Nagibina,

Bychowsky \& Nagibina, 1974: 475

Host: Jarbua terapon T. jarbua

Distribution: Gulf of Tonkin

94. P. sprostonae Bychowsky \& Nagibina, 1974

Bychowsky \& Nagibina, 1974: 479

Hosts: Deep-bodied mojarr $G$. erythrourus, slender silver-biddy G. oblongus (syn. G. macrosoma)

Distribution: Gulf of Tonkin 1974

95. P. youngi Bychowsky \& Nagibina,

Bychowsky \& Nagibina, 1974: 480

Host: Slender silver-biddy G. oblongus

Distribution: Gulf of Tonkin

Genus Triacanthinella Bychowsky \& Nagibina, 1968 1968

96. T. principalis Bychowsky \& Nagibina,

Bychowsky \& Nagibina, 1968: 154

Host: Short-nosed tripodfish Triacanthus biaculeatus

Distribution: Gulf of Tonkin

97. T. tripathii Bychowsky \& Nagibina, 1968

Bychowsky \& Nagibina, 1968: 157

Host: Long-spined tripodfish Pseudotriacanthus strigilifer

Distribution: Gulf of Tonkin

Family Diplectanidae Monticelli, 1903

Genus Calydiscoides Young, 1969 1969

98. C. flexuosus (Yamaguti, 1953) Young,

Nguyen, 2012: 36

Host: Japanese threadfin bream Nemipterus japonicus

Distribution: Ha Long bay

Genus Diplectanum Diesing, 1858

99. D. spiralis Nagibina, 1976

Nagibina, 1976: 88

Host: Dory snapper Lutjanus fulviflamma

Distribution: Gulf of Tonkin

100. D. stetoxus Nagibina, 1976

\section{Nagibina, 1976: 93}

Host: Waigieu seaperch Psammoperca waigiensis

Distribution: Gulf of Tonkin

101. D. veropolynemi Nagibina, 1976

Nagibina, 1976: 89

Host: Blackspot threadfin Polydactylus sextarius (syn. Polynemus sextarius)

Distribution: Gulf of Tonkin

Genus Laticola Yang, Kritsky, Sun, Zhang, Shi \& Agrawal, 2006

102. L. paralatesi (Nagabina, 1976) Yang, Kritsky, Sun, Zhang, Shi \& Agrawal, 2006 1976

Syn. Diplectanum paralatesi Nagabina,

Nagibina, 1976: 91

Hosts: Barramundi Lates calcarifer; blackspot threadfin Polydactylus sextarius

Distribution: Gulf of Tonkin

Genus Murraytrema Price, 1937

103. M. pricei Bychowsky \& Nagibina, 1977

Nguyen, 2012: 39

Host: Yellow drum Nibea albiflora

Distribution: Ha Long bay

Genus Murraytrematoides Yamaguti, 1958 
104. M. bychowskii (Nagibina, 1976) Oliver, 1987

Nagibina, 1976: 94

Host: Striped threadfin Polydactylus plebeius (syn. Polynemus plebeius)

Distribution: Gulf of Tonkin

Genus Paradiplectanum Domingues \& Boeger, 2008

105. P. blairense (Gupta \& Khanna, 1974) Domingues \& Boeger, 2008

Syn. Diplectanum blairense Gupta \& Khanna, 1974

Nguyen, 2012: 34

Hosts: Silver sillago Sillago sihama; Japanese sillago S. japonica

Distribution: Ha Long bay

Genus Pseudodiplectanum Tripathi, 1957

106. P. bychowskyi Nagibina, 1977

Nagibina, 1977: 86

Host: Fourlined tonguesole Cynoglossus bilineatus

Distribution: Gulf of Tonkin

107. P. caballeroi Nagibina, 1977

Nagibina, 1977: 88

Host: Tonguefish Symphurus orientalis

Distribution: Gulf of Tonkin

108. P. cynoglossum Tripathi, 1955

Nagibina, 1977: 87

Host: Fourlined tonguesole $C$. bilineatus

Distribution: Gulf of Tonkin

Genus Pseudorhabdosynochus Yamaguti, 1958

109. P. brunei Dang, Bristow, Schander \& Berland, 2013**

Dang et al., 2013: 52 bruneus

Host: Longtooth grouper Epinephelus

Distribution: Nha Trang bay

110. P. coioidesis Bu, Leong, Wong, Woo \& Foo, 1999
Hosts: Duskytail grouper E. bleekeri, malabar grouper E. malabaricus, Greasy grouper E. tauvina, warsaw grouper Hyporthodus nigritus*

Vo et al., 2010: 26

Distributions: Gulf of Tonkin, Nha Trang bay

111. P. cupatus (Young, 1969) Kritsky \& Beverley-Burton, 1986

Te, 1998: 7; Arthur \& Bui, 2006: 48

Host: sixbar grouper E. sexfasciatus

Distribution: Gulf of Tonkin

112. P. epinepheli (Yamaguti, 1938) Kritsky and Beverley-Burton, 1986

Te, 1998: 7; Arthur \& Bui, 2006: 48; Vo et al., 2010: 27

Hosts: Duskytail grouper E. bleekeri, longtooth grouper E.bruneus, malabar grouper E. malabaricus, greasy grouper $E$. tauvina, warsaw grouper Hyporthodus nigritus*

Distribution: Gulf of Tonkin

113. P. grouperi (Bu, Leong, Wong, Woo \& Foo, 1999) Wu, Li, Zhu \& Xie, 2005

Syn. Diplectanum grouperi Bu, Leong, Wong, Woo \& Foo, 1999

Vo et al., 2010: 24

Hosts: Duskytail grouper E. bleekeri, longtooth grouper E.bruneus, malabar grouper E. malabaricus, greasy grouper $E$. tauvina, warsaw grouper $H$. nigritus*

Distribution: Gulf of Tonkin

114. P. hargisi (Oliver \& Paperna, 1984)

Santos, Buchmann \& Gibson, 2000

Syn. Diplectanum hargisi Oliver and Paperna, 1984

Te, 1998: 8; Arthur \& Bui, 2006: 48

Hosts: Longtooth grouper $E$. bruneus, sixbar grouper E. sexfasciatus, greasy grouper E. tauvina

Distribution: Gulf of Tonkin

115. P. lantauensis Beverley-Burton and Suriano, 1981 
Vo et al., 2010: 28

Hosts: Duskytail grouper E. bleekeri, malabar grouper E. malabaricus, warsaw grouper Hyporthodus nigritus* bay

Distributions: Gulf of Tonkin, Nha Trang

116. P. nhatrangensis Dang, Bristow, Schander \& Berland, 2013**

Dang et al., 2013: 46

Hosts: Duskytail grouper E. bleekeri, Orange-spotted grouper $E$. coioides

Distribution: Nha Trang bay

117. P. serrani (Yamaguti, 1953) Kritsky \& Beverley-Burton, 1986

Vo et al., 2010: 32

Hosts: duskytail grouper E. bleekeri, malabar grouper E. malabaricus, greasy grouper E. tauvina, warsaw grouper Hyporthodus nigritus*

Distribution: Nha Trang bay

118. P. summanae (Young, 1969) Kritsky \& Beverley-Burton, 1986

Vo et al., 2010: 31

Hosts: duskytail grouper E. bleekeri, warsaw grouper Hyporthodus nigritus*

Distribution: Nha Trang bay

119. P. summanoides Yang, Gibson \& Zeng, 2005

Vo et al., 2010: 30

Hosts: Duskytail grouper E. bleekeri, malabar grouper E. malabaricus, warsaw grouper Hyporthodus nigritus*

Distribution: Nha Trang bay

120. P. vagamipullum (Young, 1969) Kritsky \& Beverley-Burton, 1986

Vo et al., 2010: 31

Host: Longspine grouper E. longispinis (syn. E. fario)

Distribution: Nha Trang bay

121. P. vietnamensis Dang, Bristow, Schander \& Berland, 2013**

Dang et al., 2013: 49
Hosts: Duskytail grouper E. bleekeri, orange-spotted grouper E. coioides

Distribution: Nha Trang bay

Genus Telegamatrix Ramalingum, 1955

122. T. elegans Bychowsky \& Nagibina, 1976

Bychowsky \& Nagibina, 1976: 9

Host: Pugnose ponyfish Secutor insidiator (syn. Leiognathus insidiator)

Distribution: Gulf of Tonkin

123. T. grandis Bychowsky \& Nagibina, 1976

Bychowsky \& Nagibina, 1976: 10

Host: Pugnose ponyfish $S$. insidiator

Distribution: Gulf of Tonkin

124. T. ramalingami Bychowsky \& Nagibina, 1976

Bychowsky \& Nagibina, 1976: 12

Host: Pugnose ponyfish $S$. insidiator

Distribution: Gulf of Tonkin 1937

Order GYRODACTYLIDEA Bychowsky, 1951

Family Tetraonchoididae Bychowsky,

Genus Paratetraonchoides Bychowsky, Gussev \& Nagibina, 1965

125. P. inermis Bychowsky, Gussev \& Nagibina, 1965

Bychowsky et al., 1965: 144 lebeck

Host: Longnosed stargazer Ichthyscopus

Distribution: Gulf of Tonkin

Genus Pavlovskioides Bychowsky, Gussev \& Nagibina, 1965

126. P. ichthyoscopi Bychowsky, Gussev \& Nagibina, 1965

Bychowsky et al., 1965: 146 lebeck

Host: Longnosed stargazer Ichthyscopus

Distribution: Gulf of Tonkin 
127. P. litoralis Bykhovsky, Gussev and Nagibina, 1965

Bychowsky et al., 1965: 148

Host: Snakefish Trachinocephalus myops

Distribution: Gulf of Tonkin

Genus Pseudotetraonchoides Bychowsky, Gussev \& Nagibina, 1965

128. P. bleekeriae Bychowsky, Gussev \& Nagibina, 1965

Bychowsky et al., 1965: 152

Host: sand lance Bleekeria viridianguilla

Distribution: Gulf of Tonkin 1988

Order MONOCOTYLIDEA Lebedev, 1879

Family Monocotylidae Taschenberg,

Genus Monocotyle Taschenberg, 1878

129. M. ancylostomae Timofeeva, 1984

Timofeeva, 1984: 299

Host: Bowmouth guitarfish Rhina ancylostoma

Distribution: Gulf of Tonkin

130. M. undosocirrus Timofeeva, 1984

Timofeeva, 1984: 297

Host: Twin-spot butterfly ray Gymnura bimaculata

Distribution: Gulf of Tonkin

Genus Heterocotyle Scott, 1904

131. H. armata Timofeeva, 1983

Timofeeva, 1983: 39

Host: Honeycomb stingray Dasyatis uarnak

Distribution: Gulf of Tonkin

132. H. confusa Timofeeva, 1983

Timofeeva, 1983: 37

Host: Honeycomb stingray D. uarnak

Distribution: Gulf of Tonkin

Genus Neoheterocotyle Hargis, 1955

133. N. bychowskyi (Timofeeva, 1981) Chisholm, 1994
Timofeeva, 1981: 400

Host: Giant guitarfish Rhynchobatus djiddensis (syn. Rhinobatus djiddensis)

Distribution: Gulf of Tonkin

134. N. nagibinae (Timofeeva, 1981) Chisholm, 1994

Timofeeva, 1981: 401

Host: Giant guitarfish $R$. djiddensis

Distribution: Gulf of Tonkin

135. N. rhinobatis (Pillai \& Pillai, 1976)

Chisholm \& Whittington, 1997

Timofeeva, 1981: 402

Host: Giant guitarfish $R$. djiddensis

Distribution: Gulf of Tonkin

Genus Dasybatotrema Price, 1936

136. D. spinosum Timofeeva, 1983

Timofeeva, 1983: 42

Host: Round ribbontail ray Taeniurops meyeni (Sny. Taeniura melanospilos)

Distribution: Gulf of Tonkin

Genus Timofeevia Chisholm, Wheeler \& Beverley-Burton, 1995

137. T. rajae (Timofeeva, 1983) Chisholm, Wheeler \& Beverley-Burton, 1995 1983

Syn. Dasybatotrema rajae Timofeeva,

Timofeeva, 1983: 44

Host: ray Dasyatis sp.

Distribution: Gulf of Tonkin

SUBCLASS

OLIGONCHOINEA

BYCHOWSKY, 1937 1937

Order MAZOCRAEIDEA Bychowsky, 1959

Family Allodiscocotylidae Tripathi,

Genus Allodiscocotyla Yamaguti, 1953

138. A. chorinemi Yamaguti, 1953

Lebedev, 1970a: 193; Lebedev et al., 1970: 193; Parukhin, 1976: 98 
Nguyen Manh Hung et al.

Hosts: Doublespotted queenfish Scomberoides lysan; scad Caranx sp.; scad Decapterus sp.

Distribution: Gulf of Tonkin

139. A. diacanthi Unnithan, 1962

Lebedev et al., 1970a: 193

Host: Scad Decapterus sp.

Distribution: Gulf of Tonkin

Genus Camopia Lebedev, 1970

140. C. rachycentri Lebedev, 1970

Lebedev, 1970b: 666, 1986: 151

Host: Cobia Rachycentron canadum

Distribution: Gulf of Tonkin

Genus Metacamopia (Unnithan, 1962) Lebedev, 1972

141. M. indica (Unnithan, 1962) Lebedev, 1972

Host: Doublespotted queenfish Scomberoides lysan

Lebedev, 1972b: 128; Lebedev, 1986: 153

Distribution: Gulf of Tonkin

142. M. chormemi (Yamaguti, 1953) Lebedev, 1984 153

Lebedev et al., 1970: 194; Lebedev, 1986:

Hosts: Doublespotted queenfish S. lysan; bigeye scad Selar crumenophthalmus

Distribution: Gulf of Tonkin

Family Axinidae Monticelli, 1903

Genus Loxuroides Price, 1962

143. L. pricei Nguyen, Nguyen, Bui \& Ha, 2016

Nguyen et al., 2016: 391

Host: Pharao flyingfish Cypselurus naresii

Distributions: Gulf of Tonkin; South Cental coast

Genus Unnithanaxine Price, 1962

144. U. naresii Nguyen, Nguyen, Bui \& На, 2016

Nguyen et al., 2016: 388
Host: Pharao flyingfish Cypselurus naresii

Distributions: Gulf of Tonkin; South Cental coast 1969

Family Bychowskicotylidae Lebedev,

Genus Bychowskicotyle Lebedev, 1969

145. B. plectorhynchi Lebedev, 1969

Lebedev, 1986: 99

Host: Crescent sweetlips Plectorhynchus cinctus

Distribution: Gulf of Tonkin

Genus Tonkinopsis Lebedev, 1972

146. T. transfretanus Lebedev, 1972

Lebedev, 1972a: 151

Host: Crescent sweetlips $P$. cinctus

Distribution: Gulf of Tonkin 1895

Family Diclidophoridae Cerfontaine, 1863

Genus Choricotyle van Beneden \& Hesse,

147. C. pellonae Kritsky \& Bilgee, 1973

Mamaev, 1975: 108

Host: Elongate ilisha Ilisha elongata

Distribution: Gulf of Tonkin

148. C. polynemi Mamaev, 1972

Mamaev, 1972a: 158

Hosts: Blackspot threadfin Polynemus sextarius; fourfinger threadfin Eleutheronema tetradactylum

Distribution: Gulf of Tonkin

Genus Helciferus Mamaev, 1972

149. H. tenuis Mamaev, 1972

Mamaev, 1973: 1236

Host: Osbeck's grenadier anchovy Coilia mystus

Distribution: Gulf of Tonkin

Genus Heterobothrium Cerfontaine, 1895

150. H. tonkinensis Bychowsky \& Nagibina, 1976

Bychowsky et al., 1976: 31 
Hosts: Silver-cheeked toadfish Lagocephalus sceleratus; Takifugu sp.

Distribution: Gulf of Tonkin

Genus Osphyobothrus Yamaguti, 1958 1969

151. O. bychowskyi Khoche \& Chauhan,

Mamaev \& Parukhin, 1970: 305; Parukhin, 1976: 112

Host: Greater lizardfish Saurida tumbil

Distributions: Gulf of Tonkin; South Cental coast

152. O. multivitellatus Mamaev \& Parukhin, 1970

Mamaev \& Parukhin, 1970: 308

Host: Greater lizardfish S. tumbil

Distribution: vịnh Thái Lan

Genus Papillochoricotyle Mamaev, 1975

153. P. ilishae Mamaev, 1975

Mamaev, 1975: 110

Hosts: Elongate ilisha Ilisha elongata, Ilisha sp.

Distribution: Gulf of Tonkin

Genus Upenicola Unnithan, 1966

154. U. capheni Mamaev, 1972

Mamaev, 1972b: 166

Hosts: Sulphur goatfish Upeneus sulphureus; goatfish Parupeneus sp.

Distribution: Gulf of Tonkin

155. U. digitata Mamaev, 1972

Mamaev, 1972b: 164

Hosts: Sulphur goatfish U. sulphureus; goatfish Parupeneus sp.

Distribution: Gulf of Tonkin

156. U. upeneoides Unnithan, 1966

Mamaev, 1972b: 165

Host: Sulphur goatfish U. sulphureus; goatfish Parupeneus sp.

Distribution: Gulf of Tonkin

Genus Urocotyle Unnithan, 1966

157. U. anellus Mamaev, 1972
Mamaev, 1972b: 167

Host: Sin croaker Johnius dussumieri

Distribution: Gulf of Tonkin

Family Gastrocotylidae Price, 1943

Genus Allopseudaxine Yamaguti, 1943

158. A. macrova (Unnithan, 1957) Yamaguti, 1963

Lebedev, 1968a: 49

Host: scad Caranx sp.

Distribution: Gulf of Tonkin

Genus Churavera Unnithan, 1968

159. C. triangula (Mamaev, 1967) Lebedev, 1986

Lebedev, 1986: 65

Host: Frigate tuna Auxis thazard

Distribution: Gulf of Tonkin

Genus Gastrocotyle Beneden \& Hesse, 1863

160. G. indica Subhapradha, 1951

Lebedev, 1986: 52

Host: Shrimp scad Alepes djedaba (syn. Caranx kalla)

Distribution: Gulf of Tonkin

161. G. kalla Unnithan, 1968

Lebedev, 1986: 52

Hosts: Shrimp scadA. djedaba, Blackfin scad A. melanoptera (syn. Caranx malam)

Distribution: Gulf of Tonkin

162. G. trachuri Van Beneden \& Hesse, 1863

Lebedev, 1968a: 49

Hosts: Bigeye scad Selar crumenophthalmus; scad Decapterus sp.

Distribution: Gulf of Tonkin 1890

Genus Pseudaxine Parona \& Perugia,

163. P. bivaginalis Dillon \& Hargis, 1965

Lebedev, 1986: 54

Host: Yellowstripe scad Selaroides leptolepis 
Distribution: Gulf of Tonkin

164. P. kurra Unnithan, 1968

Lebedev 1986: 54

Hosts: Torpedo scad Megalaspis cordyla; yellowtail scad Atule mate

Distribution: Gulf of Tonkin

165. P. trachuri Parona \& Perugia, 1889

Lebedev, 1986: 53

Hosts: Malabar trevally Caranoides malabaricus (syn. Caranx malabaricus); scad Caranx sp.; scad Decapterus sp.

Distribution: Gulf of Tonkin

Genus Pseudaxinoides Lebedev, 1968

166. P. bychowski Lebedev, 1977

Lebedev, 1986: 56

Host: Shrimp scad Alepes djedaba

Distribution: Gulf of Tonkin

167. P. caballeroi Lebedev, 1977

Lebedev, 1986: 56

Host: Shrimp scad A. djedaba

Distribution: Gulf of Tonkin

168. P. decapteri (Yamaguti, 1968) Lebedev, 1977

Lebedev, 1986: 57

Host: Japanese scad Decapterus maruadsi

Distribution: Gulf of Tonkin

169. P. vietnamensis Lebedev, Parukhin \& Roitman, 1970

Lebedev, 1986: 56; Lebedev et al., 1970: 198; Parukhin, 1976: 92

Hosts: Bigeye scad Selar crumenophthalmus; yellowstripe scad Selaroides leptolepis; greater amberjack Seriola dumerili; trevally Caranx sp.; scad Decapterus sp.

Distribution: Gulf of Tonkin

Genus Sibitrema Yamaguti, 1966

170. S. poonui Yamaguti, 1966

Mamaev, 1967: 994; Lebedev, 1986: 60
Hosts: Kawakawa Euthynnus affinis; frigate tuna Auxis thazard; Atlantic bluefin tuna Thunnus thynnus

Distribution: Gulf of Tonkin

Family Gotocotylidae Yamaguti, 1963

Genus Cathucotyle Lebedev, 1968

171. C. cathuaui Lebedev, 1968

Lebedev, 1968a: 46, 1970a, 1986: 143

Hosts: Narrow-barred Spanish mackerel Scomberomorus commerson, Indo-Pacific king mackerel S. guttatus

Distribution: Gulf of Tonkin

Genus Gotocotyla Ishii, 1936

172. G. acanthura (Parona \& Perugia, 1896) Meserve, 1938

Syn. G. secunda (Tripathi, 1954)

Lebedev, 1970a: 201

Host: Narrow-barred Spanish mackerel $S$. commerson

Distribution: Gulf of Tonkin

173. G. bivaginalis (Ramalingam, 1961) Rohde, 1976

Syn. G. laticauda Lebedev, 1970

Lebedev, 1970a: 202; Lebedev, 1986: 141

Host: Narrow-barred Spanish mackerel $S$. commerson

Distribution: Gulf of Tonkin

Family Heteraxinidae Unnithan, 1957

Genus Bicotyle Tripathi, 1956

174. B. perpolita Lebedev, 1968

Lebedev, 1968b : 48

Hosts: Silver pomfret Pampus argenteus; black pomfret Parastromateus niger

Distribution: Gulf of Tonkin

Genus Heteraxine Yamaguti, 1938

175. $H$. heterocerca (Goto, 1894) Yamaguti, 1938

Lebedev, 1968a: 82, 1970a: 206

Host: Bigeye scad Selar crumenophthalmus 
Distributions: Gulf of Tonkin, South Cental coast

Genus Kannaphallus Unnithan, 1957

176. K. virilis Unnithan, 1957

Lebedev, et al. 1970a: 198; Mamaev, 1990: 226

Host: Indian threadfish Alectis indica

Distribution: Gulf of Tonkin

Genus Lethrinaxine Mamaev, 1970

177. L. parva Mamaev, 1970

Mamaev, 1970: 146

Host: Grey large-eye bream Gymnocranius griseus

Distribution: Gulf of Tonkin

Genus Monaxine Unnithan, 1957

178. M. formionis Unnithan, 1957

Mamaev, 1970: 143 niger

Hosts: Black pomfret Parastromateus

Distribution: Gulf of Tonkin 1961

Family Heteromicrocotylidae Unnithan, 1953

Genus Heteromicrocotyla Yamaguti,

179. H. carangis Yamaguti, 1953

Lebedev, 1970a: 197; Mamaev, 1988a: 37

Host: Black pomfret Parastromateus niger

Distribution: Gulf of Tonkin

180. H. paruchini Mamaev, 1968

Mamaev, 1988a: 42

Hosts: Malabar trevally C. malabaricus, Carangoides sp.

Distribution: Gulf of Tonkin

181. H. polyorchis Unnithan, 1961

Lebedev, 1970a: 197

Hosts: Shadow trevally C. dinema; Bigeye trevally C. sexfasciatus

Distribu tion: Gulf of Tonkin

182. H. vaginispina Unnithan, 1961
Lebedev, 1970a: 197; Mamaev, 1988a: 43

Host: Malabar trevally C. malabaricus

Distribution: Gulf of Tonkin

Family Hexostomatidae Price, 1936

Genus Hexostoma Rafinesque, 1815

183. H. thynni (Delaroche, 1811) Rafinesque, 1815

Syn. H. auxisi Palombi, 1943

Mamaev, 1968: 18

Host: Frigate tuna Auxis thazard

Distribution: Gulf of Tonkin

Genus Neohexostoma Price, 1961 1961

184. N. euthynni (Meserve, 1938) Price,

Mamaev, 1968: 21

Hosts: Frigate tuna Auxis thazard; kawakawa Euthynnus affinis

Distribution: Gulf of Tonkin

Family Mazocraeidae Price, 1936

Genus Engraulicola George, 1960

185. E. thrissocles (Tripathi, 1959) Lebedev, 1971 1959

Syn. Paramazocraes thrissocles Tripathi,

Mamaev, 1975: 111

Host: Anchovy Thrissocles sp.

Distribution: Gulf of Tonkin

Genus Heteromazocraes Mamaev, 1981

186. H. coiliae Mamaev, 1981

Mamaev, 1981: 174 grayii

Host: Gray's grenadier anchovy Coilia

Distribution: Gulf of Tonkin

187. H. dodecacantha Mamaev, 1981

Mamaev, 1981: 176

Host: Anchovy Thrissocles sp.

Distribution: Gulf of Tonkin 1981 
Nguyen Manh Hung et al.

Mamaev, 1975: 112, 1981: 177

Hosts: Anchovy Thrissocles hamilton's thryssa T. hamiltonii

Distribution: Gulf of Tonkin

Genus Leptomazocraes Mamaev, 1975

189. L. orientalis (Chauhan, 1950) Mamaev, 1975

Mamaev, 1975: 103

Host: Slender rainbow sardine Dussumieria elopsoides

Distribution: Gulf of Tonkin

190. L. trispina (Unnithan, 1964) Mamaev, 1981

Mamaev, 1981: 179

Host: Slender rainbow sardine $D$. elopsoides

Distribution: Gulf of Tonkin

Genus Mazocraeoides Price, 1936

191. M. dussumieri Mamaev, 1975

Mamaev, 1975: 104

Host: Slender rainbow sardine $D$. elopsoides

Distribution: Gulf of Tonkin

192. M. prashadi Chauhan, 1950

Mamaev, 1975: 105

Hosts: Sardinella Amblygaster sp.

Distribution: Gulf of Tonkin

Genus Neomazocraes Price, 1943

193. N. dorosomatis (Yamaguti, 1938) Price, 1943

Nguyen, 2012: 40 thrissa

Host: Chinese gizzard shad Clupanodon

Distribution: Ha Long bay

Genus Pseudoanthocotyle Bychowsky \& Nagibina, 1954

194. P. pavlovskyi Bychowsky \& Nagibina, 1954

Bychowsky \& Nagibina, 1954: 32
Host: Indian mackerel Rastrelliger kanagurta

Distribution: Gulf of Tonkin

Genus Pseudomazocraes Price, 1961

195. P. longicauda (Mamaev, 1975) Mamaev, 1984

Mamaev, 1975: 113

Host: Slender rainbow sardine Dussumieria elopsoides

Distribution: Gulf of Tonkin 1879

Family Microcotylidae Taschenberg,

Genus Caballeraxine Lebedev, 1972

196. C. chainanica (Lebedev, Parukhin \& Roitman, 1970) Lebedev, 1972

Lebedev, et al. 1970: 198; Mamaev \& Kurochin, 1976: 18

Host: Malabar trevally Caranx malabaricus

Distribution: Gulf of Tonkin

Genus Diplostamenides Unnithan, 1971 1986

Syn. Microcotyle hemiatriospinalis Lebedev, Parukhin and Roytman, 1970

Lebedev, 1970a: 196; Lebedev, et al. 1970: 171; Parukhin, 1976: 122; Mamaev, 1986: 201

Host: Amberjacks Seriola sp.

Distribution: Gulf of Tonkin

Genus Intracotyle Mamaev, 1970

198. I. orientalis Mamaev, 1970

Mamaev, 1970: 184

Host: Saddle grunt Pomadasys maculatus

Distribution: Gulf of Tonkin

Genus Lutianicola Lebedev, 1970

199. L. haifonensis Lebedev, 1970

Lebedev, 1970a: 194

Host: Emperor red snapper Lutjanus sebae, russell's snapper $L$. russellii 
Distribution: Gulf of Tonkin

Genus Microcotyle Van Beneden \& Hesse, 1863

200. M. arrips Sandars, 1945

Bychowsky \& Nagibina, 1975b: 215

Hosts: Whitecheek monocle bream Scolopsis vosmeri, lattice monocle bream $S$. taeniopterus; yellowtail amberjack Seriola lalandi (syn. S. aureovittata)

Distribution: Gulf of Tonkin

201. M. fistulariae Mamaev, 1989

Mamaev, 1989: 143

Host: Red cornetfish Fistularia petimba

Distribution: Gulf of Tonkin

Genus Monomacracanthus Mamaev, 1976

202. M. cadodai Mamaev, 1976

Mamaev, 1976: 31

Hosts: Large yellow croaker Larimichthys crocea (syn. Pseudosciaena crocea); sin croaker Johnius dussumieri

Distribution: Gulf of Tonkin

Genus Polylabris Euzet \& Cauwet, 1967

203. P. gerres (Sandars, 1944) Mamaev \& Parukhin, 1976

Mamaev \& Parukhin, 1976: 250

Hosts: Saddleback silver-biddy Gerres lucidus; whipfin silver-biddy G. filamentosus

Distribution: Gulf of Tonkin

204. P. tubicirrus (Paperna \& Kohn, 1964) Mamaev \& Parukhin, 1976

Mamaev \& Parukhin, 1976: 247

Hosts: Toothpony G. minuta; pugnose ponyfish L. insidiator

Distribution: Gulf of Tonkin

Genus Polylabroides Mamaev \& Parukhin, 1976

205. P. zini Mamaev, 1987

Mamaev, 1988b: 196

Hosts: Goldsilk seabream A.berda, Yellowfin seabream A. latus
Distribution: Gulf of Tonkin

Genus Polynemicola Unnithan, 1971

206. P. ambigua Mamaev, 1977

Mamaev, 1977: 94

Hosts: Blackspot threadfin Polynemus sextarius, Eleutheronema sp.

Distribution: Gulf of Tonkin

207. P. sciaenae Mamaev, 1977

Mamaev, 1977: 96

Host: Sin croaker Johnius dussumieri

Distribution: Gulf of Tonkin

Genus Tonkinaxine Lebedev, Parukhin \& Roitman, 1970

208. T.homocerca Lebedev, Parukhin and Roytman, 1970

Lebedev, 1970a: 197; Lebedev et al., 1970a: 175; Parukhin, 1976: 86

Hosts: Blackbanded trevally Seriolina nigrofasciata; greater amberjack Seriola dumerili, Seriola sp.

Distributions: Gulf of Tonkin, South Cental coast

Family Paramonaxinidae Mamaev, 1990

Genus Incisaxine Mamaev, 1970

209. I. dubia Mamaev, 1970

Mamaev, 1970: 149

Host: Whipfin silver-biddy Gerres filamentosus

Distribution: Gulf of Tonkin 1903

Family Plectanocotylidae Monticelli, 1972

Genus Triglicola Mamaev \& Parukhin,

210. T. tonkinensis Mamaev \& Parukhin, 1972

Mamaev \& Parukhin, 1972: 66; Parukhin, 1989: 121

Host: Gurnard Lepidotrygla sp.

Distribution: Gulf of Tonkin 
Family Protomicrocotylidae Johnston \& Tiegs, 1922

Genus Bilaterocotyloides Ramalingam, 1961

211. B. carangis Ramalingam, 1961 124

Lebedev et al., 1970: 194; Parukhin, 1989:

Host: Torpedo scad Megalaspis cordyla

Distribution: Gulf of Tonkin

212. B. madrasensis Radha, 1966

Lebedev, 1986: 115

Host: Torpedo scad M. cordyla

Distribution: Gulf of Tonkin

Genus Neomicrocotyle Ramalingam, 1960

213. N. indica Ramalingam, 1960

Bychowsky \& Nagibina, 1975b: 216

Host: Bigeye trevally Caranx sexfasciatus

Distribution: Gulf of Tonkin

Genus Vallisiopsis Subhapradha, 1951

214. V. contorta Subhapradha, 1951

Lebedev, 1986: 118

Host: False trevally Lactarius lactarius

Distribution: Gulf of Tonkin

215. V. sphyraenae Yamaguti, 1968

Lebedev, 1986: 118

Host: Yellowtail barracuda Sphyraena flavicauda (syn. S. langsar)

Distribution: Gulf of Tonkin

Genus Youngiopsis Lebedev, 1972 1972

216. Y. australis (Young, 1968) Lebedev,

Lebedev, 1972b: 132

Host: Obtuse barracuda S. obtusata

Distribution: Gulf of Tonkin

Family

Yamaguti, 1965

Pseudodiclidophoridae

Genus Quillonella Lebedev \& Parukhin, 1970 1970

217. Q. ventrosa Lebedev \& Parukhin,

Lebedev \& Parukhin, 1970: 458

Hosts: Cobia Rachycentron canadum; Japanese scad Decapterus maruadsi

Distributions: Gulf of Tonkin, South Cental coast

Family Pterinotrematidae Caballero \& Bravo-Hollis, 1955

Genus Pterinotrema Caballero, BravoHollis \& Grocott, 1954

218. P. mirabile Bychowsky \& Nagibina, 1959

Bychowsky \& Nagibina, 1959: 218

Host: Bonefish Albula vulpes

Distributions: Gulf of Tonkin, South Cental coast

Family Thoracocotylidae Price, 1936

Genus Pricea Chauhan, 1945

219. P. multae Chauhan, 1945

Lebedev, 1970: 201

Hosts: Narrow-barred Spanish mackerel Scomberomorus commerson, Indo-Pacific king mackerel S. guttatus

Distributions: Gulf of Tonkin, South Cental coast 1963

Genus Pseudothoracocotyla Yamaguti,

220. P. ovalis (Tripathi, 1956) Yamaguti, 1963

Lebedev, 1970: 204

Hosts: Narrow-barred Spanish mackerel Scomberomorus commerson, Indo-Pacific king mackerel S. guttatus

Distributions: Gulf of Tonkin, South Cental coast

Notes: “*”: The distribution of the warsaw grouper Hyporthodus nigritus is mainly in the western of Atlantic Ocean, so all records of warsaw grouper Hyporthodus nigritus in the off Vietnam could be misidentified. The correct species, for instance, is generally considered to a tenspine grouper Epinephelus 
exsul, which is distributed in Indo-Pacific region; “**”: Not accepted yet by WoRMS (2020).

\section{DISCUSSION}

In this paper, we updated the list of monogenea fauna from marine fishes of Vietnam. The list includes 220 species, this number is greater comparing with 135 species in the list of Arthur \& Bui (2006). In addition, the modification of contemporary classifications of several taxa also was updated.

At higher taxonomic levels, i.e. subclass, there are two classification systems which are bilateral used at the present (Wheeler \& Chisholm, 1995), including (1) the system was proposed by Llewellyn (1970) and revised by Beverley-Burton (1984) and (2) the system was proosed by Bychowsky (1937) and revised by Boeger \& Kritsky (1993). The classification system of Llewellyn (1970) and Beverley-Burton (1984) is divided the class of Monogenea into two subclasses, Monopisthocotylea and Polyopisthocotylea, based on several morphological characters of fluke, e.g. the structure, shape and size of opisthohaptor and parasitic position on its hosts. This system is simple and is used by WoRMS (2020) for updating data. The system of Bychowsky (1937) and Boeger \& Kritsky (1993) divides the class of Monogenoidea into three subclasses, Polyonchoinea, Polystomatoinea and Oligonchoinea. This sytem is more complicated to compare to the system of Llewellyn (1970) and BeverleyBurton (1984), but it has more comprehensive views, and was revised basing on 141 character states in 47 homologous series and employing phylogenetic systematics (Boeger \& Kritsky, 1993). Therefore we used the classification system of Bychowsky (1937) and Boeger \& Kritsky (1993), and updated data of lower taxa by using WoRMS (2020). The bilateral using two classification systems are not contradiction, because almost taxonomic levels at family and under family are the same. Monogenean fauna in Vietnam is belonging to two subclasses Polyonchoinea and Oligonchoinea.
At the level of genus, Allopseudaxine was moved to Axinidae from Gastrocotylidae (Hayward \& Rohde, 1999a), Tonkinaxine was moved to Microcotylidae from Heteraxinidae (Mamaev, 1986). Mamaev (1990) transfered some genera from Microcotylidae to other families, e.g. Incisaxine was moved to Paramonaxinidae, Kannaphallus and Lethrinaxine were moved to Heteraxinidae.

At the level of species, Arthur \& Bui (2006) arranged two species, Vallisia chorinemi and Triglicola tonkinensis, in Anthocotylidae, however, they are belonging to Allodiscocotylidae (Lebedev, 1984) and Plectanocotylidae (Parukhin 1989), separately. In addition, Lebedev (1984) also transferred $V$. chorinemi to Metacamopia. Bychowsky \& Nagibina (1970) moved Ancyrocephalus bilobatus and A. spinicirrus to Haliotrema; similarly, A. scapulasser was transferred to Protogyrodactylus (Gussev, 1973), and Diplectanum hargisi was moved to Pseudorhabdosynochus (Santos et al., 2000). Moreover, the name of four species, Dawesia incise, Microcotyle hemiatriospinalis, Gotocotyla laticaudata và $G$. secunda were changed in succession to Pseudothoracocotyla ovalis, Diplostamenides sciaenae, Gotocotyla bivaginalis and G. Acanthura (Hayward \& Rohde, 1999b; Mamaev, 1986; Rohde, 1976).

Three species, Pseudorhabdosynochus bruneus, $P$. nhatrangensis and $P$. vietnamensis which were found from grouper by Dang et al. (2013), are unaccepted because the publication is not compliant with Article 8.5 (2012) of the International Commission on Zoological Nomenclature WoRMS (2020). In this paper, however, we listed all three mentioned species because of theirs exist.

In addition, there are 23 monogenean species, which were identified at genus level, including: 17 species of Ligophorus (Garasev et al., 2011a, b; Dmitrieva et al., 2013); 1 species of Diplectanum (Mamaev, 1970); 1 species of Gastrocotyle (Lebedev, 1970a); 1 species of Microcotyle (Lebedev et al., 1970); 1 species of Pseudaxine (Parukhin, 1976); 1 species of Benedenia (Te, 1998); 1 species of Haliotrema (Vo et al., 2010). All of them did 
not count in the list above but the future studies should clarify the taxonomic position of these species.

Relating to the host-parasite relationships, the great majority of monogeneans (70,9\%, 156/220 species) use just only one host species, it shows the specialization of monogeneans to choose theirs host. Other monogenea species were found on two or even seven host species, e.g. Haliotrema epinepheli has been found on the gills of 7 marine fish species. About fish hosts, some species are only infected by only one monogenean species while other species are infected by many congeneric monogenean species, e.g, Spotted scat Scatophagus argus, or by monogeneans of diferent genera, e.g. groupers Epinephelus spp., snapper Lutjanus spp.

In conclusion, the monogenean fauna from marine fish of Vietnam is quite diverse, with 220 recoded species. However, almost period studies have only forcused to discorvery the biodiversity of these flukes. There are currently no studies on genetic, biological, ecological characters of monogeneans in the off Vietnam. In addition, the pathogens, which caused by monogeneans, also need to further study for reducing the damage to aquaculture.

\section{REFERENCES}

Arthur J. R., Bui Q. T., 2006. Checklist of the parasites of fishes of Vietnam. FAO Fisheries Technical Paper 369/2, 133 pp.

Beverley-Burton M., 1984. Monogenea and Turbellaria. In Margolis L., \& Kabata Z. (eds.), Guide to the parasites of fishes of Canada, Part 1, p. 5-209. Canadian Spec. Publ. Fish. Aquat. Sci. 74.

Boeger W. A., Kritsky D. C., 1993. Phylogeny and a revised classification of the Monogenoidea Bychowsky, 1937 (Platyhelminthes). Syst. Parasitol. 26: 1-32. https://doi.org/10.1007/ BF00009644.

Bychowsky B. E., 1937. Ontogenesis and phylogenetic interrelationships of parasitic flatworms. lzvestiya Akademiya Nauk SSSR, Set. Biologiya, 4, 1353-1383. (in Russian: English translation, 1981, Gloucester Point, Virginia: Virginia Institute of Marine Science, Translation Series no. 26).

Bychowsky B. E., Gussev A. V., Nagibina L. F., 1965. Monogenetic trematodes of family Tetraonchoididae Bychowsky, 1951. Trudy Zoologicheskogo Instituta, 53: 140-166. (in Russian).

Bychowsky B. E., Mamaev Yu. L., Nagibina L. F., 1976. Revision of the genus Heterobothrium Cerfontaine, 1895 (Diclidophoridae). Trudy BiologoPochvennogo Instituta, 34 (137): 29-39. (in Russian).

Bychowsky B. E., Nagibina L. F., 1954. On a new representative of marine monogenetic trematodes from the Pacific Ocean. Zoologicheskii Zhurnal, 33: 30-38. (in Russian).

Bychowsky B. E., Nagibina L. F., 1959. On new representatives of monogenetic trematodes from The South China Sea. Acta Zoologica Sinica, 11, 211-236. (in Chinese).

Bychowsky B. E., Nagibina L. F., 1967. New Capsalidae (Monogenoidea) from Pacific fishes. Parazitologiya, 1: 521-528. (in Russian).

Bychowsky B. E., Nagibina L. F., 1968. Triacanthinella, a new genus of marine monogenean parasites of hornfishes. Parazitologiya, 2: 148-157. (in Russian).

Bychowsky B. E., Nagibina L. F., 1969a. Ancyrocephalinae (Dactylogyridae, Monogenoidea) from fishes of the family Ariidae. Parazitologiya, 3: 357-368. (in Russian).

Bychowsky B. E., Nagibina L. F., 1969b. New genus of monogeneans of the subfamily Ancyrocephalinae (Dactylogyridae). Parazitologiya, 3: 518527. (in Russian).

Bychowsky B. E., Nagibina L. F., 1970a. Ancyrocephalinae

(Monogenoidea, 
Dactylogyridae) from the sea fishes of the family Pomadasyidae. Anales del Instituto de Biología Univetsidad Nacional Autónoma de México. 41. Ser. Zoologia Num Unico., 19-28.

Bychowsky B. E., Nagibina L. F., 1970b. On the new and little known species of the genus Haliotrema Johnston \& Tiegs, 1922 (Monogenoidea). Zoologicheskii Zhurnal, 49: 1789-1801. (in Russian).

Bychowsky B. E., Nagibina L. F., 1971. New and little known species of the genus Haliotrema Johnston \& Tiegs, 1922 (Monogenoidea). Zoologicheskii Zhurnal, 50: 25-40. (in Russian).

Bychowsky B. E., Nagibina L. F., 1974. New species of Protogyrodactylus (Dactylogyridae, Ancyrocephalinae). Parazitologiya, 8: 473-483. (in Russian).

Bychowsky B. E., Nagibina L. F., 1975a. New data about genus Ancyrocephaloides Yamaguti, 1938 (Dactylogyridae, Ancyrocephalinae). In K. K. Tiwari, C. B. L. Srivastava, \& R. B. S. Chauhan (Eds.), Commemorative volume (pp. 68-73). Orissa: Zoological Society of India.

Bychowsky B. E., Nagibina L. F., 1975b. New data on the postembrional development of some members of Monogenoidea. Parazitologiya, 9: 209-219. (in Russian).

Bychowsky B. E., Nagibina L. F., 1976. New species of the genus Telegamatrix Ramalingam, 1955 (Monogenoidea, Diplectanidea). Biologiya Morya, Vladivostok, 2: 10-15. (in Russian).

Bychowsky B. E., Nagibina L. F., 1977. On systematic position of some monogeneans of marine fishes. Parazitologicheskii Sbornik, 27: 5-17. (in Russian).

Dang B. T., Bristow G. A., Schander C., Berland B., 2013. Three new species of Pseudorhabdosynochus (Monogenea: Diplectanidae) from Vietnamese grouper (Epinephelus spp.) (Perciformes: Serranidae). Int $J$ Aquatic Science, 4: 44-58.
Dang T. B., Levsen A., Schander C., Bristow G. A., 2010. Some Haliotrema (Monogenea: Dactylogyridae) from cultured grouper (Epinephelus spp.) with emphasis on the phylogenetic position of Haliotrema cromileptis. J Parasitol., 96: 30-39. https://doi.org/10.1645/GE-2140.1.

Dmitrieva E. V., Garasev P. I., Kolpakov N. V., Nguyen V. H., Ha D. N., 2013. On monogeneans (Plathelminthes, Monogenea) fauna of marine fishes in Vietnam. III. Ligophorus spp. from species of mullets (Pisces, Mugilidae). Bulletin of the Pacific Ocean Fisheries Scientific Research Center, 172: 224-236. (in Russian].

Dmitrieva E. V., Vodiasova E., Ermolenko A., Nguyen V.H., Ha D.N., Nguyen M. H., 2018. Morphological and molecular characteristic of Ligophorus fenestrum (Platyhelminthes: Monogenea) from new host-Planiliza melinopterus (Actinopterygii: Mugilidae) in the Gulf of Tonkin off Vietnam. Contemporary Parasitology-Major Trends and Challenges. Proceedings of the VI Congress of the Society of Parasitologists, Russia: International Conference, October 15-19, Saint Petersburg.

Egorova T. P., 2000. New monogeneans from the genus Dionchus (Capsalidae: Dionchinae). Parazitologiia, 34: 252-258. (in Russian).

Egorova T. P., Korotaeva V. D., 1990. Trochopus antigoniae sp. n. (Monogeneoidea: Capsalidae) from the fishes of the South China Sea. Parazitologiya, 24: 442-446. (in Russian).

Garasev P. I., Dmitrieva E. V., Ha D. N., Kolpakov N. V., Nguyen V. H., 2011a. On monogeneans (Plathelminthes, Monogenea) fauna of marine fishes in Vietnam. I. Ligophorus spp. from Valimugil engeli Bleeker (Pisces, Mugilidae). Bulletin of the Pacific Ocean Fisheries Scientific Research Center, 165: 196-208. (in Russian). 
Garasev P. I., Dmitrieva E. V., Ha D. N., Kolpakov N. V., Nguyen V. H., 2011b. On monogeneans (Plathelminthes, Monogenea) fauna of marine fishes in Vietnam. II. Ligophorus spp. from Valimugil seheli (Forsska ${ }^{\circ}$ ) (Pisces, Mugilidae). Bulletin of the Pacific Ocean Fisheries Scientific Research Center, 165: 209-215. (in Russian).

Gussev A. V., 1967. New species of Dactylogyrus from fishes of the Far East. Parazitologicheskii Sbornik, 23: 250-254. (in Russian).

Gussev A. V., 1973. Revision of the genus Protogyrodactylus (Monogenoidea) and a description of the new species $P$. marinus. Folia Parasitol., 20: 17-27.

Gussev A. V., 1985. Order Dactylogyridea. In: O.N. Bauer (Ed.) Key to parasites of freshwater fish of the fauna of the USSR. Vol. 2. Parasitic metazoans. Leningrad: Nauka, pp. 15-251. (in Russian).

Hayward C.J., Rohde K., 1999a. Revision of the monogenean family Gotocotylidae (Polyopisthocotylea). Invertebrate Taxonomy, 13: 425-460. https://doi.org/10.1071/it98003.

Hayward C. J., Rohde K., 1999b. Revision of the monogenean subfamily Thoracocotylinae Price, 1936 (Polyopisthocotylea: Thoracocotylidae), with the description of a new species of the genus Pseudothoracocotyla Yamaguti, 1963. Syst. Parasitol., 44: 157-169. https://doi.org/10.1023/A:1006236713378.

Kritsky D. C., Boeger W. A., 2002. Neotropical Monogenoidea. 41. New and previously described species of Dactylogyridae (Platyhelminthes) from the gills of maine freshwater perciform fishes (Teleostei) with proposal of a new genus and a hypothesis on phylogeny. Zoosystema, 24: 7-40.

Kritsky D. C., Nguyen H. V., Ha N. D., Heckmann R. A., 2016. Revision of Metahaliotrema Yamaguti, 1953 (Monogenoidea: Dactylogyridae), with new and previously described species from the spotted scat Scatophagus argus (Linnaeus) (Perciformes: Scatophagidae) in Vietnam. Syst Parasitol., 93: 321-335. https://doi.org/10.1007/s11230-015-9621-9.

Lebedev B. I., 1968a. On the helminthofauna of carangid fishes of the Pacific Ocean. p. 80-85. In Gelminty Dalnego Vostoka $\mathrm{i}$ Tikhogo Okeana. Soobshch. Dalnevost. Filiala Imeni V.L. Komarova, Sibirskoe Otdelenie, Akad. Nauk SSSR, 26, Vladivostok. (in Russian).

Lebedev B. I., 1968b. Monogenea of fishes of the New Zealand-Australian Shelf and the South China Sea (Monogenoidea, Gasterocotylidae, Gasterocotylinae). p. 46-55. In Gelminty Zhivotnykh Tikhogo Okeana. Izd. "Nauka", Moscow. (in Russian).

Lebedev B. I., 1970a. Helminths of epipelagic fishes of the South China Sea. In: Oshmarin, P.G., Mamaev, Yu.L. \& Lebedev, B.I. (Eds) Helminths of animals of Southeast Asia. Moscow: Nauka, pp. 191-208. (in Russian).

Lebedev B. I., 1970b. The substantiation of a new family of monogenetic flukes (Monogenoidea) and the description of Camopia rachycentri gen. et sp. n. Zoologicheskii Zhurnal, 49: 665-672. (in Russian).

Lebedev B. I., 1972a. The new family of higher monogeneans - Calyxinellidae fam. n. (Monogenoidea). Trudy BiologoPochvennogo Instituta, Vladivostok, 11: 148-153. (in Russian).

Lebedev B. I., 1972b. The taxonomy of monogeneans of suborder Gastrocotylinea. In: Investigations on the Fauna, Systematics, and Biochemistry of Helminths in the Far East. Proceedings of the Far-East Science Centre, USSR Academy of Sciences 11: 121-145. (in Russian).

Lebedev B. I., 1984. Parasites of plants and animals. In: B. I. Mamaev, B. A. Dboritki, Erozenko. Academy of Sciences of the 
USSR, Institute of Biology and Edafology of Central Asia and Far East. Vladivostok, 108 pp. [In Russian].

Lebedev B. I., 1986. Monogenoidea, Suborder Gastrocotyline. Leningrad: Nauka, 200pp. [In Russian].

Lebedev B. I., Parukhin A. M, 1970. A new monogenoid, Quilonella ventrosa gen. \& sp. n. and their position in the system. Parazitologiya, 4: 458-465. [In Russian].

Lebedev B. I., Parukhin A. M., Roitman V. A., 1970. Monogenetic trematodes, Oligongchoinea (Monogenoidea), parasites of horse mackerel fishes of North Vietnam Gulf. Biologya Morya, Kiev, 20: 167-187. [In Russian].

Llewellyn J., 1970. Monogenea. In: Technical reviews: taxonomy, genetics and evolution of parasites. Second Interna tional Congress of Parasitology. J Parasitol., 56, Sect. II, Pt. 3, 493-504. https://doi.org/10.2307/3277585.

Mamaev Yu. L., 1967. Pseudaxine triangula sp.n.and Metapseudaxine ventrosicula gen. \& sp. n. and their position in the system of Monogenoidea. Zoologicheskii Zhurnal, 46: 993-998. [In Russian].

Mamaev Yu. L., 1968. Helminths of tunas of the South China Sea. In: Skrjabin K. I., Manaev Yu. L. (Eds) Helminths of the Pacific Ocean. Moscow: Nauka, pp. 5-27. [In Russian].

Mamaev Yu. L., 1969. A new genus of monogeneans of the family Calceostomatidae (Parona \& Perugia, 1890). Parazitologicheskii Sbornik, 24: 169-172. [In Russian].

Mamaev Yu. L., 1970. Helminths of some food fishes of the Gulf Tonkin. In: Oshmarin, P.G., Manaev, Yu.L. \& Lebedev, B.I. (Eds) Helminths of animals of Southeast Asia. Moscow: Nauka, 121-190. [In Russian].

Mamaev Yu. L., 1972a. The description of a new monogenean from the subfamily Choricotylinae, with some notes about genus Choricotyle compostition. Trudy
BiologoPochvennogo Instituta, Vladivostok, 11 (114): 155-162. [In Russian].

Mamaev Yu. L., 1972b. The monogeneans of the genus Upenicola Unnithan, 1966. Trudy Biologo-Pochvennogo Instituta, Vladivostok, 11 (114): 163-169. [In Russian].

Mamaev Yu. L., 1973. A new species and genus Helciferus tenuis (Diclidophoridae) from fishes of the family Engraulidae. Zoologicheskii Zhurnal, 52: 1235-1238. [In Russian].

Mamaev Yu. L., 1975. Monogeneans of clupeiform fishes from the South China Sea (Mazocridae and Diclidophoridae). Trudy Biologo-Pochvennogo Instituta, Vladivostok, 26 (129): 102-114. [In Russian].

Mamaev Yu. L., 1976. A new representative of microcotylid monogeneans, Monomacracanthus cadodai gen. \& sp. nov. Biologiya Morya, 3: 30-33. [In Russian].

Mamaev Yu. L., 1977. The monogeneans of the genus Polynemicola Unnithan, 1971. Trudy Biologo-Pochvennogo Instituta, Vladivostok, 47 (150): 89-100. [In Russian].

Mamaev Yu. L., 1981. Some new monogenean species and genera of the family Mazocraeidae. Helminthologia, 18: 169-187.

Mamaev Yu. L., 1986. The taxonomical composition of the family Microcotylidae Taschenberg, 1879 (Monogenea). Folia Parasitol., 33, 199-206.

Mamaev Yu. L., 1988a. Monogeneans of the family Heteromicrocotylidae Unnithan, 1916. Trudy Zoologicheskogo Instituta, Leningrad, 177: 35-48. [In Russian].

Mamaev Yu. L., 1988b. Polylabroides zini sp. n., a new species of higher monogenean from a marine fish, Acanthopagrus latus. Helminthologia, 25: 195-200. 
Nguyen Manh Hung et al.

Mamaev Yu. L., 1989. On species composition and morphological features of the Microcotyle genus (Microcotylidae, Monogenoidea). In: Lebedev, B.I. (Ed.) Investigation in parasitology, collection of papers. Vladivostok: DVNTs SSSR, 167 pp. [In Russian].

Mamaev Y. L., 1990. The systematical composition of the family Heteraxinidae and other allied families of Monogenea. Folia Parasitol. 37: 225-230.

Mamaev Yu. L., Kurochin Yu. V., 1976. Monogeneans Caballeraxine chainaica (Leb., Par. \& Roitm. 1970), their mode of attachment to host, morphology and systematic position. Biologiya Morya, Vladivostok, 2: 16-21. [In Russian].

Mamaev Yu. L., Parukhin A. M., 1970. Monogeneans of the genus Osphyobothrus Yamaguti, 1958 (Monogenoidea, Diclidophoridae). Parazitologiya, 6: 503-311. [In Russian].

Mamaev Yu. L., Parukhin A. M., 1972. Monogeneans of the family Plectanocotylidae Poche, 1926 new representatives of the subfamily Plectanocotylinae. Parazitologiya, 6: 65-74. [In Russian].

Mamaev Yu. L., Parukhin A. M., 1976. Genus Polylabris Euzet et Cauwet, 1967 and some related species of microcotylids (Monogenoidea: Microcotylidae). Parazitologiya, 10: 245-254. [In Russian].

Nagibina L. F., 1968. Bychowskya drepane, a new genus and species of the family Calceostomatidae Parazitologiya, 2: 289.

Nagibina L. F., 1976. New species of the genus Diplectanum (Monogenoidea, Diplectanidae). Trudy BiologoPochvennogo Instituta, 35 (138): 89-96. [In Russian].

Nagibina L. F., 1977. New data on the genus Pseudodiplectanum Tripathi, 1955 (Monogenoidea, Diplectanidae). Exerta parasitologica en memoria del Doctor
Eduardo Caballero y

Caballero.Universidad Nacional Autonóma de México. Instituto de Biologia Publicaciones Especiales, 4: 85-89.

Nguyen H. M., Nguyen H. V., Bui T. N., Ha N. D., 2016. Two new axinid species (Monogenea: Axinidae) from the Pharao flyingfish Cypselurus naresii (Günther) (Beloniformes: Exocoetidae) in the Gulf of Tonkin off Vietnam. Syst Parasitol, 93: 387-394. https://doi.org/10.1007/s11230016-9635-y.

Nguyen V. H., 2012. Helminth fauna of marine fishes in Halong Bay. PhD thesis., Thai Nguyen University, 136 pp. [In Vietnamese].

Parukhin A. M., 1976. Parasitic worms of food fishes from the southern seas. Akademiya Nauk Ukrainskoi SSR, Ordena Trudovogo Krasnogo Zhameni Institut Biologii Yuzhnykh Morei im. A. O. Kobalevskogo, Izdatelstvo "Naukova Dumka,” Kiev, 182 pp. [In Russian].

Parukhin A. M., 1989. Parasitic worms of bottom fishes from the southern seas. Akademiya Nauk Ukrainskoi SSR, Institut Biologii Yuzhnykh Morei im. A. O. Kobalevskogo, Izdatelstvo "Naukova Dumka,” Kiev, 154 p. [In Russian].

Santos C. P., Buchmann K., Gibson D. I., 2000. Pseudorhabdosynochus spp. (Monogenea: Diplectanidae) from the gills of Epinephelus spp. in Brazilian waters. Syst. Parasitol., 45: 145-153 (2000). Te B. Q., 1998. Parasites and fish diseases of grouper cage culture in Ha Long Bay. Scientific Reports, Research Institute for Aquaculture No. 1, 14 p. [In Vietnamese].

Timofeeva T. A., 1981. New species of the genus Horricauda (Monogenea, Monocotylidae) Parazitologiya, 15: 399-406. [In Russian].

Timofeeva T. A., 1983. New representatives of monocotylids (Monogenea, Monocotylidae) from cartilaginous fishes of the South China and Yellow Seas. Trudy Zoologicheskogo Instituta, Leningrad, 181: 35-47. [In Russian]. 
Timofeeva T. A., 1984. New members of monocotylids of the genus Monocotyle and Merizocotyle from rays of the South China and Yellow Seas (Monogenea, Monocotylidae). Parazitologiya, 18: 296-305. [In Russian].

Timofeeva T. A., 1989. New species of Dionchus genus from South China and Yellow Seas (Monogenoidea, Dionchidae). In: Lebedev, B.I. (Ed.) Investigations in parasitology, collection of papers. Vladivostok: DVNTs AN SSSR, pp. 55-66. [In Russian].

Timofeeva T. A., 2000. New monogeneans of genus Dionchus (Capsalidae: Dionchinae). Parazitologiya, 34: 252-258.
Vo T. D., Bristow G. A., Nguyen H. D., Vo T. D., Nguyen N. T. N., 2010. The parasites of grouper and sea bass in Vietnam. Ho Chi Minh: Agriculture Publishing House, $178 \mathrm{pp}$.

Wheeler T. A., Chisholm L. A., 1995. Monogenea versus Monogenoidea: the case for stability in nomenclature. Syst. Parasitol., $\quad 30(3)$ : 159-164. https://doi.org/10.1007/BF00010466.

WoRMS, 2020. Pseudorhabdosynochusbrunei Dang, Bristow, Schander \& Berland, 2013. Accessed at: http://www.marinespecies.org/aphia.php? $\mathrm{p}=$ taxdetails\&id $=834007$ on 2020-02-24. 